
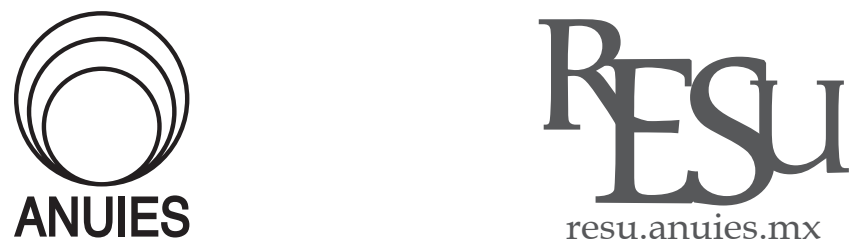

ARTí́CULO

\title{
La experiencia universitaria. Análisis de factores motivacionales y sociodemográficos
}

\author{
The university experience. Analysis of motivational and \\ sociodemographic factors
}

\author{
Luis Doña Toledo y Teodoro Luque Martínez * \\ *Universidad de Granada, Granada, España. \\ Correos electrónicos: tluque@ugr.es, luisdt@ugr.es
}

Recibido el 22 de enero del 2018; aceptado el 03 de julio del 2019

\section{Resumen}

En el presente trabajo se analiza el impacto de las motivaciones de elección de los estudios, el sexo, el área científica y el nivel educativo de los padres. La muestra está integrada por 9360 egresados. Los resultados indican que las motivaciones de tipo intrínsecas, sobretodo la vocación, conducen a una mayor satisfacción, variando según el área científica. Las mujeres son más vocacionales y tienen una mayor satisfacción. Los alumnos cuyos padres tienen bajo nivel de estudios tienen una mejor valoración de la experiencia universitaria. Además, a través de la técnica de segmentación jerárquica se determina el perfil de egresado que presenta una mayor satisfacción. Conocer la importancia de las variables preuniversitarias y su impacto en la satisfacción tras finalizar los estudios es una información fundamental para orientar las decisiones de las instituciones universitarias, de los futuros estudiantes y de la administración educativa.

Palabras clave: Educación superior; Satisfacción; Motivos de elección; Nivel de estudios de los padres; Egresados. 


\begin{abstract}
In this paper, the impact of the motivational choice to study, sex, scientific area and educational level of parents is analyzed.. The sample consists of 9360 graduates. The results indicate that intrinsic type motivations -vocation- lead to greater satisfaction varying according to the scientific area. Women are more vocational and have greater satisfaction. Students whose parents have low education have a better assessment of the college experience. In addition, the hierarchical segmentation technique determines the profile of graduates with greater satisfaction. Knowing the importance of pre-university variables and their impact on satisfaction after studies conclusion is a key to guide universities decisions, prospective students and educational management information.
\end{abstract}

Keywords: Higher education; Satisfaction; Motivations; Education level of parents; Students.

\title{
Introducción
}

L a universidad desempeña un papel fundamental como motor de la sociedad por su papel en la formación, la innovación, el desarrollo, el empleo o el bienestar social. Según la Organización para la Cooperación y el Desarrollo Económicos (OCDE, 2014), existe una relación positiva entre el nivel de educación de la sociedad, en general, y la salud, la calidad de vida, la felicidad o la satisfacción de los integrantes de un país.

El sector de la educación superior está en continuo proceso de cambio para su modernización y su adaptación. Los principales desafíos o retos de la universidad derivan de nuevos escenarios y agentes además de la aparición de alternativas educativas. Así hay un aumento de la demanda con diversificación por edad y necesidades tanto territoriales como sociales, mayores niveles de internacionalización, nuevos procesos (Open Learning o la aparición de los MOOC), reestructuración de la oferta docente, así como la necesidad de afrontar desafíos económicos, financieros o tecnológicos, además de la formación de espacios universitarios, como ha sido la aparición del Espacio Europeo de la Educación Superior, entre otras iniciativas (Neave y Veiga, 2013; Espinosa-López, 2015; Luque et al., 2015).

Estas transformaciones afectan a todo el proceso de enseñanza-aprendizaje universitario, desde el inicio, al identificar las necesidades y los objetivos que debe atender la enseñanza universitaria, hasta el final, al comprobar si se cumplen las expectativas y si la experiencia ha sido satisfactoria. En este sentido, hay un protagonista especial: el estudiante egresado, que es en quien se centra este estudio. Una vez realizado todo el proceso de elección de estudios, experimentado la etapa universitaria y sus consecuencias en cuanto a inserción laboral y desarrollo personal, su valoración aporta un incentivo a tener muy en cuenta 
como guía para posibles reformas o innovaciones. En ese proceso de decisión inciden variables personales como motivaciones (Faye y Sharpe, 2008), o sexo (Sáinz et al., 2004) y sociales como familia o nivel de estudio de los padres (Byrne et al., 2012).

El objetivo es analizar los diferentes motivos -identificando los más importantes - por los cuales un estudiante decide realizar determinados estudios, acudir a la universidad como apuesta de futuro laboral y "de vida"; estudiar cómo sus características personales y sociales condicionan esas decisiones y, en particular, cómo inciden en su satisfacción final.

\section{Factores en el proceso de evaluación del estudiante universitario}

En el proceso de evaluación del estudiante universitario intervienen diferentes tipos de factores. Unos son de índole macro, como los contextos económico, social o político, a niveles regional e internacional, que operan a modo de marco general en el que se desarrolla el proceso de enseñanza-aprendizaje.

Un segundo tipo de factores es de tipo intermedio, se refieren al sistema universitario en su conjunto, y a las universidades concretas que lo forman. Cada universidad tiene una reputación, una determinada oferta, una localización, un contexto y todo un historial de actuaciones presentes y pasadas que condicionan su elección, sin olvidar la importancia de la cartera de títulos o tipo de estudios ofrecidos que, cuando es claramente vocacional, predomina sobre la influencia que pueda tener la universidad.

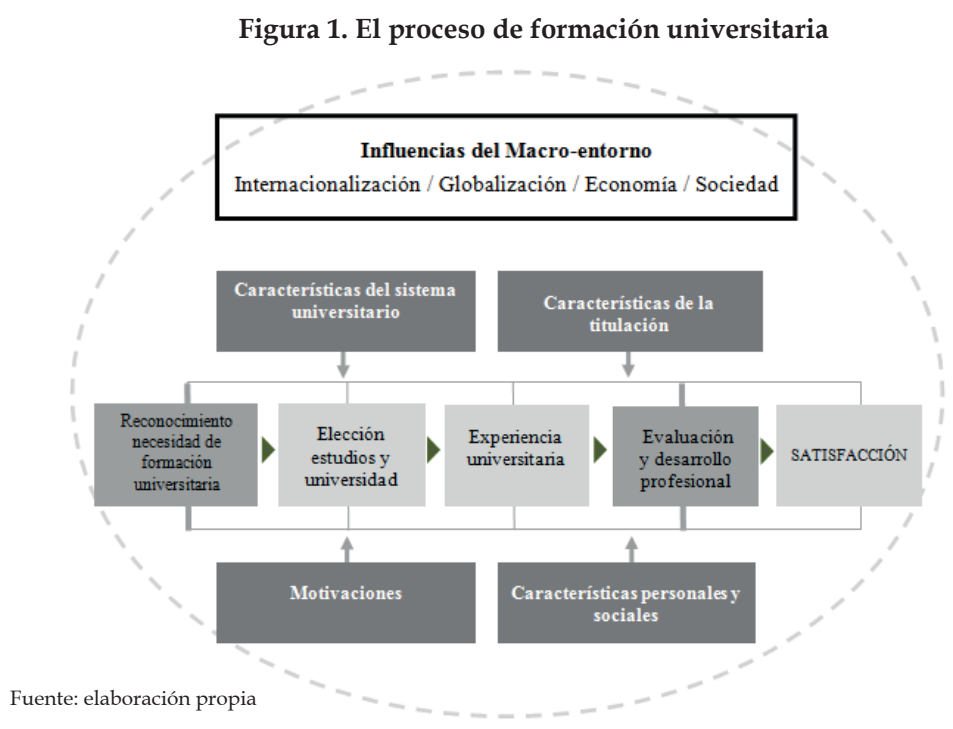


Finalmente, el tercer tipo de factores hace referencia a las características individuales y sociales del estudiante. Es decir, los referidos a sus gustos, preferencias, motivaciones, actitudes, y demás características psicológicas, pero también los relativos a la familia, grupo social o contexto económico al que pertenece.

La evaluación que realiza el estudiante que termina sus estudios viene determinada por todo ese conjunto de factores. Este estudio se centra en el análisis de las motivaciones en general, pero también su importancia, habida cuenta de otras características sociales y personales tan determinantes como el área científica elegida, el sexo y el nivel de formación de los padres.

\section{Motivación}

A toda acción le precede una motivación. La motivación conduce a un objetivo, pero esto puede concretarse en diferentes comportamientos. La búsqueda de una mejor formación para un mejor desarrollo personal y laboral puede desembocar en la elección o no de estudios universitarios, para posteriormente elegir una universidad (Escolano-Pérez, 2014).

Para Faye y Sharpe (2008), muchos de los estudios sobre la motivación académica se encuadran dentro de la teoría de la autodeterminación. Dentro de este marco, Deci y Ryan (2000) diferencian comportamientos motivados extrínsecamente, como un medio para alcanzar un fin deseable y que se realiza por placer o satisfacción, asumiendo que el concepto motivacional dominante es un objetivo más que una necesidad. Precisamente, éste es el caso del candidato a estudiante universitario, pues la motivación surge por objetivos tanto de desarrollo personal como laboral. Faye y Sharpe (2008) establecen dos tipos principales de motivos académicos:

- Motivos intrínsecos: los alumnos pueden tener iniciativas personales o una vocación determinada aislada de su entorno inmediato. Entran en juego los propios gustos e intereses y el deseo de crecer y desarrollarse intelectualmente, además de acceder a un puesto de trabajo que les enriquezca.

- Motivos extrínsecos: los estudiantes pueden estar influenciados por un ambiente concreto, un aprendizaje previo o un entorno familiar determinado. La decisión que efectúa un joven está condicionada por factores como la oferta de plazas, la nota obtenida o las limitaciones sociales o económicas. También destaca la influencia del entorno, por ejemplo, de la actual crisis económica, que puede determinar su elección.

Una misma conducta puede estar impulsada por diversidad de motivos tanto intrínsecos como extrínsecos. La interacción entre motivación intrínseca y 
extrínseca tiene efectos variados. Cuando hay una conducta motivada de forma intrínseca, la intervención de motivos extrínsecos tendrá efectos negativos sobre la motivación futura, tal y como demuestran diferentes estudios (Condry, 1987).

En la tabla I pueden observarse los motivos extrínsecos e intrínsecos tratados por la literatura.

La literatura es relativamente abundante en el análisis de las motivaciones extrínsecas e intrínsecas, pero no tanto en comprobar la relación de esas motivaciones con la satisfacción tras la experiencia universitaria y la incorporación al trabajo. Esta relación es tratada por Paolillo y Estes (1982), Solinas et al. (2012) y Law y Yuen (2012), quienes establecieron que los motivos intrínsecos conducen a una mayor valoración de la etapa universitaria. De acuerdo con la teoría de la autoconfirmación de Deci y Ryan (2000) y Faye y Sharpe (2008), cabría pensar que perseguir un objetivo llevado por una motivación intrínseca, salvo experiencias muy negativas en la universidad, reforzaría la satisfacción. Todo esto conduce a las siguientes propuestas de hipótesis:

H1a: Al decidir el tipo de estudio, hay diferencias en la importancia de los motivos extrínsecos y los motivos intrínsecos. Siendo estos últimos más tenidos en cuenta.

H1b: Quienes eligieron por motivos intrínsecos presentarán mayor nivel de satisfacción que quienes lo hicieron por motivos extrínsecos.

Tabla 1.

Motivos analizados en la literatura.

\begin{tabular}{|c|c|c|c|c|c|c|c|c|c|c|c|}
\hline Autores & 胥 & 苟 & 莺 & 品 & 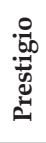 & पें & 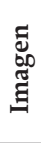 & $\underset{\mathscr{E}}{\stackrel{\dot{E}}{\mathscr{D}}}$ & 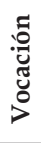 & $\begin{array}{l}\vec{\Xi} \\
\dot{\Xi} \\
\stackrel{\Xi}{J}\end{array}$ & $\begin{array}{l}\text { D } \\
\stackrel{x}{0}\end{array}$ \\
\hline \multicolumn{12}{|l|}{ Sewell y Shah (1968) } \\
\hline \multicolumn{12}{|l|}{ Paolillo y Estes (1982) } \\
\hline \multicolumn{12}{|l|}{ Rivas (1990) } \\
\hline \multicolumn{12}{|l|}{ Gul et al.(1992) } \\
\hline \multicolumn{12}{|l|}{ Adams et al. (1994) } \\
\hline \multicolumn{12}{|l|}{ Felton et al.(1995) } \\
\hline \multicolumn{12}{|l|}{ Paulsen y Gentry (1995) } \\
\hline \multicolumn{12}{|l|}{ Sirvanci (1996) } \\
\hline \multicolumn{12}{|l|}{ Ahmed et al. (1997) } \\
\hline \multicolumn{12}{|l|}{ Auyeung y Sands (1997) } \\
\hline \multicolumn{12}{|c|}{ Broekemier y Seshadri (1999) } \\
\hline \multicolumn{12}{|l|}{ Tang et al.(1999) } \\
\hline \multicolumn{12}{|l|}{ Paa y McWhirter (2000) } \\
\hline Gámez y Marrero (2003) & & & & & & & & & & & \\
\hline
\end{tabular}

Continúa... 


\begin{tabular}{|c|c|c|c|c|c|c|c|c|c|c|c|}
\hline Autores & 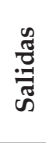 & 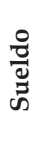 & 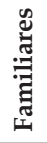 & 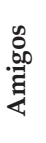 & 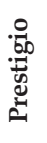 & பี & 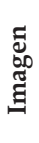 & 芯 & 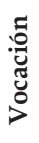 & $\begin{array}{l}\vec{\Xi} \\
\dot{\Xi} \\
\dot{U}\end{array}$ & 胥 \\
\hline \multicolumn{12}{|l|}{ Price, et al. (2003) } \\
\hline \multicolumn{12}{|l|}{ Byrne y Flood (2005) } \\
\hline \multicolumn{12}{|l|}{ González (2005) } \\
\hline \multicolumn{12}{|l|}{ Myburgh (2005) } \\
\hline \multicolumn{12}{|c|}{ Drewes y Michael (2006) } \\
\hline \multicolumn{12}{|c|}{ Jackling y Calero (2006) } \\
\hline \multicolumn{12}{|c|}{ Mansfield y Warwick (2006) } \\
\hline \multicolumn{12}{|l|}{ Maringe (2006) } \\
\hline \multicolumn{12}{|l|}{ Martín y Salas (2006) } \\
\hline \multicolumn{12}{|c|}{ Shanka, Quintal y Taylor (2006) } \\
\hline \multicolumn{12}{|l|}{ Tan y Laswad (2006) } \\
\hline \multicolumn{12}{|l|}{ Byrne y Flood (2007) } \\
\hline \multicolumn{12}{|l|}{ Vrontis et al.(2007) } \\
\hline \multicolumn{12}{|l|}{ Castro y Egaña (2008) } \\
\hline \multicolumn{12}{|l|}{ Arquero et al. (2009) } \\
\hline \multicolumn{12}{|c|}{ Sugahara Y Boland (2009) } \\
\hline \multicolumn{12}{|l|}{ Palos y Drobot (2010) } \\
\hline \multicolumn{12}{|l|}{ Zellweger et al. (2011) } \\
\hline \multicolumn{12}{|c|}{ Byrne, Willis y Burke (2012) } \\
\hline \multicolumn{12}{|l|}{ Gatica et al. (2012) } \\
\hline \multicolumn{12}{|c|}{ Joseph, Mullen y Spake (2012) } \\
\hline \multicolumn{12}{|l|}{ Law y Yuen (2012) } \\
\hline \multicolumn{12}{|c|}{ López Bonilla et al. (2012) } \\
\hline \multicolumn{12}{|l|}{ Solinas et al. (2012) } \\
\hline \multicolumn{12}{|l|}{ Wardley et al. (2013) } \\
\hline Luque et al. (2015) & & & & & & & & & & & \\
\hline
\end{tabular}

Fuente: elaboración propia

\section{Área científica}

La mayoría de las investigaciones revelan que los estudiantes eligen principalmente su carrera con base en el convencimiento de que es acorde con su forma de ser y sus capacidades (Rivas, 1990). No obstante, la literatura demuestra diferencias en las motivaciones según el área de conocimiento. Por ejemplo, Ahmed et al. (1997) estudiaron la influencia del entorno y la voca- 
ción para elegir la carrera del área empresarial donde los aspectos intrínsecos apenas tienen importancia siendo las salidas profesionales y las recomendaciones de los padres los principales motivos alcanzado resultados similares a Auyeung y Sands (1997).

Byrne y Flood (2005), Byrne, Willis y Burke (2012) demostraron que la elección de la carrera es un compendio compuesto por ampliar conocimientos, el futuro profesional y la influencia parental y de los amigos. Byrne et al. (2012), en el caso del área empresarial, encontraban que los principales aspectos eran la influencia parental (en consonancia con los estudios de Law y Yuen, (2012) y las salidas profesionales, mientras que en el resto de áreas de conocimiento eran más importante los factores intrínsecos.

Paolillo y Estes (1982) realizaron un estudio comparativo donde los médicos y los ingenieros presentaban un mayor nivel de vocación, la mayoría influenciados por su entorno familiar y la ocupación laboral de sus padres. Los abogados eran los más preocupados por las salidas profesionales y la remuneración económica. Wardley et al. (2013), en un estudio longitudinal entre el primer y el último año de universidad, demostraban que aquellos motivados intrínsecamente mostraban una mayor implicación y unos mejores resultados académicos. En general, la mayoría de la literatura se ha centrado en los motivos de elección sin considerar la mediación del área científica, aunque se localizan algunos (ver Tabla 2).

Tabla 2.

Estudios comparativos entre motivaciones y áreas de conocimiento

\begin{tabular}{|c|c|c|}
\hline Autor/Año & Área de conocimiento & Principales conclusiones \\
\hline Paolillo y Estes (1982) & $\begin{array}{l}\text { Ingeniería, } \\
\text { medicina, derecho y } \\
\text { contabilidad. }\end{array}$ & $\begin{array}{l}\text { En Salud y Técnicas influye vocación y } \\
\text { tradición familiar, en Sociales las salidas } \\
\text { profesionales. }\end{array}$ \\
\hline García et al. (1994) & $\begin{array}{l}\text { Ciencias de la Salud, } \\
\text { Experimentales, } \\
\text { Sociales, Humanas y } \\
\text { Económico-Jurídicas. }\end{array}$ & $\begin{array}{l}\text { Mayor importancia de motivos extrínsecos } \\
\text { en alumnos de Sociales y Económico- } \\
\text { Jurídicas. }\end{array}$ \\
\hline Paulsen y Gentry (1995) & $\begin{array}{l}\text { Finanzas y } \\
\text { contabilidad } \\
\text { (Ciencias Sociales). }\end{array}$ & $\begin{array}{l}\text { En los estudiantes de finanzas, las salidas } \\
\text { profesionales es el principal motivo. }\end{array}$ \\
\hline Ahmed et al. (1997) & Ciencias Sociales. & $\begin{array}{l}\text { Mayor importancia de los aspectos } \\
\text { extrínsecos en el campo de las Ciencias } \\
\text { Empresariales y notable influencia paterna } \\
\text { en la elección. }\end{array}$ \\
\hline Broekemier y Seshadri (1999) & Todas & $\begin{array}{l}\text { Influencia de los padres, que otorgan } \\
\text { una mayor importancia a los aspectos } \\
\text { académicos y salidas profesionales. }\end{array}$ \\
\hline Tang, Fouad y Smith (1999) & $\begin{array}{l}\text { Ingeniería, } \\
\text { psicología, derecho, } \\
\text { empresariales, física } \\
\text { e informática. }\end{array}$ & $\begin{array}{l}\text { Gran influencia familiar como motivo de } \\
\text { elección (aunque no con ello la tradición } \\
\text { familiar). Los alumnos suelen sentirse } \\
\text { presionados ante padres que no acudieron } \\
\text { a la universidad. }\end{array}$ \\
\hline
\end{tabular}




\begin{tabular}{|c|c|c|}
\hline Autor/Año & Área de conocimiento & Principales conclusiones \\
\hline Gámez y Marrero (2003) & $\begin{array}{l}\text { Derecho, biología y } \\
\text { psicología. }\end{array}$ & $\begin{array}{l}\text { Gran importancia de alcanzar cierto logro } \\
\text { y prestigio profesional y de la tradición } \\
\text { familiar e influencia de amigos. }\end{array}$ \\
\hline Zeegers (2004) & $\begin{array}{l}\text { Ciencias de la Salud } \\
\text { y Experimentales. }\end{array}$ & $\begin{array}{l}\text { Quienes eligieron por vocación alcanzan } \\
\text { mejores resultados académicos y muestran } \\
\text { una mayor implicación. }\end{array}$ \\
\hline Byrne y Flood (2005) & Ciencias Sociales & $\begin{array}{l}\text { La motivación es el resultado de una } \\
\text { combinación de factores intrínsecos } \\
\text { e intrínsecos (salidas profesionales y } \\
\text { enriquecimiento intelectual). }\end{array}$ \\
\hline González (2005) & Todas & $\begin{array}{l}\text { La vocación influye en todas las áreas. Las } \\
\text { salidas profesionales en el área de Ciencias } \\
\text { Sociales y la tradición familiar en Ciencias } \\
\text { de la Salud. }\end{array}$ \\
\hline Byrne y Flood (2007) & $\begin{array}{l}\text { Contabilidad vs } \\
\text { Ciencias Sociales. }\end{array}$ & $\begin{array}{l}\text { Contabilidad tiene una mayor influencia } \\
\text { parental así como de la vocación. }\end{array}$ \\
\hline Castro y Egaña (2008) & Todas & $\begin{array}{l}\text { Humanidades elige por la vocación } \\
\text { mientras que Ciencias Sociales y } \\
\text { Enseñanzas Técnicas están motivadas por } \\
\text { la tradición familiar y el futuro laboral. }\end{array}$ \\
\hline Arquero et al.(2009) & Empresas & $\begin{array}{l}\text { Los estudiantes están motivados } \\
\text { principalmente por las salidas } \\
\text { profesionales y el desarrollo intelectual. }\end{array}$ \\
\hline Wardley et al (2013) & Todas & $\begin{array}{l}\text { Los factores extrínsecos (retribución, } \\
\text { profesión) ejercen una mayor influencia. }\end{array}$ \\
\hline Luque et al. (2015) & Todas & $\begin{array}{l}\text { El principal motivo es la vocación seguido } \\
\text { por el de salidas profesionales y el de } \\
\text { completar y ampliar conocimientos. }\end{array}$ \\
\hline
\end{tabular}

Fuente: Elaboración propia.

A partir de estos estudios establecemos las siguientes hipótesis:

H2a: La presencia de las motivaciones es diferente por áreas científicas.

H2b: Hay diferencias del nivel de satisfacción por áreas científicas según las motivaciones.

H2c: Dentro de una misma área científica, las motivaciones de tipo intrínseco provocarán mayor satisfacción que las de tipo extrínseco.

\section{Sexo}

La literatura presenta evidencias de que existe una clara diferenciación por sexo en la elección de estudios (Lafuente, 2004; Sáinz et al., 2004; Mingo, 2016). Los motivos que subyacen a la elección son diferentes para ambos sexos: ellas eligen normalmente buscando la satisfacción de sus intereses o la 
preferencia personal, y los hombres se guían por las oportunidades profesionales o la remuneración (Muñoz y Mullet, 1990; Duart et al., 1993). Este conflicto de rol de las mujeres que se enfrentan a las condiciones (establecidas por hombres) del mundo laboral puede explicar, en parte, las dificultades para el desarrollo profesional de las mujeres $y$, por lo tanto, sus motivos de elección universitaria pues condiciona el futuro laboral (Eccles et al., 1999; Zeegers, 2004).

López Sáez (1995) analizó las diferencias en la elección de carreras típicamente femeninas o masculinas. La única diferencia entre hombres y mujeres era que los primeros tenían más en cuenta la posible retribución futura. Sáinz et al. (2004) determinaron que alrededor del 90\% de las mujeres en carreras típicamente femeninas (como psicología, enfermería, pedagogía o magisterio) mencionaban la vocación y los factores intrínsecos a la profesión, como la autorrealización o la necesidad de ayudar a los demás. Por el contrario, las mujeres que estudian carreras típicamente masculinas (ingenierías) aludían a aspectos más instrumentales, como el salario o la proyección profesional.

En definitiva, los hombres suelen guiarse en mayor medida por los aspectos extrínsecos y las mujeres por motivos intrínsecos (Duart et al., 1993, López-Bonilla et al., 2012). Así se propone que:

H3a: Las motivaciones de elección de estudios son diferentes por sexo.

H3b: El nivel de satisfacción general tras la finalización de estudios difiere por sexo según las motivaciones.

H3c: Para personas del mismo sexo, las motivaciones intrínsecas provocan mayor nivel de satisfacción.

\section{Nivel de formación de los padres}

Este es un factor que tiene una especial relevancia porque informa del entorno próximo (Palos y Drobot, 2010; Gatica et al., 2012). La influencia que puedan ejercer los progenitores tiene mucho que ver con su nivel de formación, además esto afecta hábitos, comportamientos y resultados académicos.

La transición de la secundaria a la universidad es una etapa compleja donde los padres ocupan un lugar destacado en el conjunto de los referentes que participan en la decisión del estudiante. Los distintos grupos de referencia (amigos, maestros, personas significativas, etc.) juegan un papel fundamental en la toma de decisiones de un individuo desde el punto de vista psicológico (Phillips et al., 2001).

Silverstone y Williams (1979) encontraron que el 26\% de los estudiantes consideró la influencia de los padres como factor de elección de carrera. Flint 
(1992) y Allen (2004) concluían que esta influencia explica el 35\% de la elección universitaria y que aquellos estudiantes cuyos padres tenían altos niveles de estudio alcanzaban un mejor expediente académico. Por el contrario, Hearn (1998) concluía que los padres apenas influyen.

Especialmente en familias con padres graduados universitarios, el entorno del hogar se convierte en un lugar vital de información y acceso a estudios superiores (McDonough, 1997). La existencia de una tradición familiar de asistir a la universidad puede ser, a priori, una forma de aumentar el deseo de sentirse integrado en su red social o dentro de su grupo de referencia. El éxito académico de los padres influye en la propia biografía educativa de los hijos (Schnabel et al., 2002). No obstante, también se constata un mayor éxito educativo de estudiantes cuyos padres cuentan con bajos niveles educativos (Ceja, 2006). En estos casos ejerce una gran influencia el deseo de mejorar, de responder al esfuerzo de los padres y de conseguir un mejor estatus como respuesta al esfuerzo realizado para la formación de sus hijos.

En general, la literatura constata que los alumnos que tienen progenitores con estudios superiores alcanzan mejores resultados académicos y que tienen una mayor reafirmación con su elección de acudir a la universidad. Por tanto:

H4a: Las motivaciones de elección son diferentes según el nivel de formación de los padres.

H4b: El nivel de satisfacción general difiere por el nivel de formación de los padres según las motivaciones.

H4c: Dentro de un mismo nivel de formación de los padres, las motivaciones intrínsecas provocan mayor nivel de satisfacción.

\section{Método}

\section{Trabajo de campo}

Para llevar a cabo el análisis se utilizaron los tres últimos informes del estudio de egresados de la Universidad de Granada (Luque et al., 2012, 2013, 2014). Esto supone una amplia muestra que permite establecer análisis conjunto de diferentes oleadas de graduados.

La encuesta se administraba unos tres años después de que los alumnos terminasen sus grados. Con ello se pretende medir el impacto de la experiencia posuniversitaria en la valoración, la utilidad y la percepción de sus estudios y de la institución. Los datos se recogieron en tres períodos diferentes 
desde 2012 a 2014. El contacto con los egresados se realizaba principalmente mediante correo electrónico.

El cuestionario constaba de cinco partes: (1) Experiencia universitaria de grado; (2) Experiencia universitaria de posgrado; (3) Experiencia laboral; (4) Adecuación de los estudios con el trabajo; (5) Características sociodemográficas.

\section{Muestra}

Desde el año 2009 al 2011 han finalizado los estudios en la Universidad de Granada 22.829 alumnos de grado de los cuales se ha podido obtener una muestra de 9.380 egresados (41.1\%). En la tabla 3 se ofrece en detalles las características de la muestra donde se ofrecen los datos de las cinco áreas científicas que recoge el sistema universitario español y la distribución según el nivel de formación de los padres.

Tabla 3. Descripción de la muestra $(\mathrm{N}=9.360)$

\begin{tabular}{|c|c|c|c|}
\hline \multirow{2}{*}{ Variable } & \multirow{2}{*}{ Categoría } & \multicolumn{2}{|c|}{ Muestra } \\
\hline & & $\mathbf{N}$ & $\%$ \\
\hline \multirow{2}{*}{ Género } & Hombre & 3.307 & 35.3 \\
\hline & Mujer & 6.073 & 64.7 \\
\hline \multirow{3}{*}{ Año de finalización } & 2009 & 2.535 & 27.0 \\
\hline & 2010 & 3.515 & 37.5 \\
\hline & 2011 & 3.330 & 35.5 \\
\hline \multirow{2}{*}{ Trabaja } & Sí & 5.444 & 58.0 \\
\hline & No & 3.839 & 41.4 \\
\hline \multirow{5}{*}{ Área científica } & Ciencias de la Salud & 1.114 & 11.9 \\
\hline & Ciencias Experimentales & 1.052 & 11.2 \\
\hline & Ciencias Sociales & 4.719 & 50.3 \\
\hline & Enseñanzas Técnicas & 1.304 & 13.9 \\
\hline & Humanidades & 1.191 & 12.7 \\
\hline \multirow{3}{*}{ Estudios de los padres } & Sin estudios/primarios & 3.374 & 36.6 \\
\hline & Secundarios & 2.229 & 24.2 \\
\hline & Superiores & 3.608 & 39.2 \\
\hline
\end{tabular}

Fuente: Elaboración propia. 
Ante la variedad de motivaciones, se ha optado por centrar el análisis en las más relevantes según la literatura. Como motivaciones intrínsecas se utilizan la vocación y ampliar-completar estudios, y como extrínsecas: salidas profesionales, recomendación de terceras personas, imposibilidad de elegir otra opción por no cumplir requisitos y tradición-recomendación familiar.

Como áreas científicas se han utilizado las cinco que se reconocen en el sistema universitario español y que se detallaban en la tabla. En cuanto al nivel de formación de los padres se ha distinguido tres categorías también indicado en la tabla.

La satisfacción general se midió mediante una escala mono-ítem de 1 a 5 . La media general de satisfacción alcanza una puntuación de 3.7.

\section{Resultados}

\section{Motivos de elección y satisfacción}

La motivación más tenida en cuenta en la elección de estudios fue la vocación, ya que más de la mitad de los encuestados (57.3\%) afirmaron estar influenciados por este motivo intrínseco. Es el único que aparece en más de la mitad de la muestra. A distancia sigue el primer motivo extrínseco, la motivación de las salidas profesionales (30\%).

Tabla 4. Porcentaje sobre total según motivos de elección (pueden seleccionarse más de un motivo)

\begin{tabular}{lll}
\hline Motivos intrínsecos & Elección & $\%$ \\
\hline Vocación & Sí & 57.50 \\
\hline Ampliar y completar conocimientos & Sí & 17.30 \\
\hline Motivos extrínsecos & Sí & 29.50 \\
\hline Salidas profesionales & Sí & I9.50 \\
\hline Recomendación de otras personas & Sí & 6.00 \\
\hline Imposibilidad de elegir la opción deseada & Sí & 7.10 \\
\hline Tradición familiar & & \\
\hline
\end{tabular}

La tercera motivación es también de tipo intrínseco, "completar y ampliar conocimientos" (17.3\%). Los otros motivos extrínsecos considerados tienen 
una presencia menor en la elección, sin alcanzar el 10\% de los casos. Se comprueba que las motivaciones tienen diferente importancia en la decisión de elección del estudiante y que motivaciones intrínsecas tienen una mayor presencia. Por tanto, se encuentra apoyo empírico para la hipótesis H1a.

Se comprueba mediante anova la existencia de diferencias estadísticamente significativas en las motivaciones intrínsecas. Los que eligieron por estos motivos presentan mayor nivel de satisfacción. Por el contrario, no se constatan diferencias estadísticamente significativas en el nivel de satisfacción general por las motivaciones extrínsecas, a excepción de quienes eligieron por imposibilidad que presentan menor nivel de satisfacción.

Tabla 5. Motivos de elección y satisfacción general (entre paréntesis nivel de significación de las diferencias)

\begin{tabular}{llll}
\hline Motivos intrínsecos & Elección & $\begin{array}{c}\text { Media } \\
\text { Satisfacción }\end{array}$ & Desv. típica \\
\hline \multirow{2}{*}{ Vocación $(0,00)$} & Sí & 3.73 & 0.98 \\
\cline { 2 - 4 } & No & 3.61 & 0.98 \\
\hline \multirow{2}{*}{ Ampliar y completar conocimientos } & Sí & 3.78 & 0.93 \\
\cline { 2 - 4 } & No & 3.66 & 0.99 \\
\hline
\end{tabular}

Motivos extrínsecos

\begin{tabular}{lccl}
\hline \multirow{2}{*}{ Salidas profesionales (n.s.) } & Sí & 3.66 & 0.96 \\
\cline { 2 - 4 } Recomendación de otras personas & No & 3.69 & 0.99 \\
\hline \multirow{2}{*}{ Imposibilidad de elegir la opción deseada } & $\mathrm{Si}$ & 3.70 & 0.97 \\
\cline { 2 - 4 } & $\mathrm{No}$ & 3.68 & 0.98 \\
\hline \multirow{2}{*}{ Tradición familiar } & $\mathrm{Si}$ & 3.50 & 1.00 \\
\hline & $\mathrm{No}$ & 3.69 & 0.97 \\
\cline { 2 - 4 } & $\mathrm{Si}$ & 3.61 & 0.97 \\
\hline
\end{tabular}

La elección por motivo vocacional es lo que marca la diferencia en la satisfacción, siempre provoca mayor nivel de satisfacción. Lo mismo ocurre al comparar el segundo motivo intrínseco (ampliar estudios o formación) con las salidas profesionales. En consecuencia, se encuentra apoyo empírico para la H1b.

Estos resultados coinciden con las conclusiones de los estudios realizados por Paulsen y Gentry (1995), Zeegers (2004) o Wardley et al. (2013), entre otros, ya que los motivos intrínsecos producen una mayor valoración en los estudiantes dado el carácter psicológico de realizar los estudios con los que realmente disfruta el alumno. 


\section{Motivaciones de elección y área científica}

Todas las motivaciones, tanto intrínsecas como extrínsecas presentan diferencias estadísticamente significativas por áreas científicas, como suele mostrar la literatura analizada. Entre las primeras, la vocación destaca más en los campos de Humanidades y Ciencias de la Salud, y la de ampliar-completar conocimientos en Humanidades y Experimentales. Entre las extrínsecas, las salidas profesionales destacan en Técnicas, Ciencias de la Salud y algunas titulaciones de Sociales.

La recomendación de terceros se consideró más en Técnicas y Experimentales. Quienes no pudieron hacer sus estudios preferidos como primera opción destacan más en Experimentales y Ciencias de la Salud. Los resultados son similares a otros estudios realizados (Paa y McWhirter, 2000; Myburgh, 2005; Sugahara y Boland, 2009). Mientras que la tradición-recomendación familiar predomina más en Ciencias de la Salud y Enseñanzas Técnicas, en consonancia con Paolillo y Estes (1982) y González (2005). Por tanto, se encuentra apoyo empírico a la H2a formulada.

Tabla 6. Motivos de elección por área científica (porcentaje sobre el total) y media de satisfacción por área científica

\begin{tabular}{lllllll}
\hline $\begin{array}{l}\text { Motivos } \\
\text { intrínsecos }\end{array}$ & Elección & $\begin{array}{c}\text { Ciencias } \\
\text { Experi- } \\
\text { mentales }\end{array}$ & $\begin{array}{c}\text { Humani- } \\
\text { dades }\end{array}$ & $\begin{array}{c}\text { Ciencias } \\
\text { Sociales }\end{array}$ & $\begin{array}{c}\text { Ciencias } \\
\text { Salud }\end{array}$ & $\begin{array}{c}\text { Ense- } \\
\text { nanzas } \\
\text { Técnicas }\end{array}$ \\
\hline Vocación (0.00) & Sí & 57.7 & 70.1 & 52.8 & 66.2 & 55.8 \\
\hline $\begin{array}{l}\text { Ampliar y completar } \\
\text { conocimientos (0.00) }\end{array}$ & Sí & 19.4 & 25.6 & 18.8 & 7.9 & 10.4 \\
\hline $\begin{array}{l}\text { Motivos } \\
\text { extrínsecos }\end{array}$ & Sí & 20.4 & 12.8 & 27.8 & 31.0 & 57.1 \\
\hline $\begin{array}{l}\text { Salidas profesionales } \\
(0.00)\end{array}$ & Sí & 10.5 & 6.7 & 9.8 & 8.3 & 11.1 \\
\hline $\begin{array}{l}\text { Recomendación de } \\
\text { otras personas (0.01) }\end{array}$ & Sí & 9.7 & 2.4 & 6.2 & 8.2 & 4.1 \\
\hline $\begin{array}{l}\text { Imposibilidad de elegir } \\
\text { la opción deseada } \\
(0.00)\end{array}$ & Sí & 3.3 & 4.3 & 7.2 & 10.7 & 9.4 \\
\hline $\begin{array}{l}\text { Tradición familiar } \\
(0.00)\end{array}$ & Media & 3.72 & 3.72 & 3.67 & 3.81 & 3.56 \\
\hline \begin{tabular}{l} 
Satisfacción $(\mathbf{0 . 0 0 )}$ \\
\hline
\end{tabular} & & & & & \\
\hline
\end{tabular}


Por otro lado, también se constatan diferencias significativas en la satisfacción por áreas, alcanzando el mayor nivel los que estudiaron Ciencias de la Salud frente a los que estudiaron Enseñanzas Técnicas. Pero la cuestión principal es qué ha ocurrido dentro de un mismo campo $(\mathrm{p}<0.05)$ :

- En Experimentales, quienes eligieron estudios que no eran su primera opción muestran menor nivel de satisfacción.

- En Humanidades presentan menor nivel de satisfacción quienes siguieron la recomendación familiar frente a los que no, y quienes no tuvieron en cuenta las salidas profesionales frente a los que sí.

- En Ciencias de la Salud claramente están más satisfechos los que eligieron por motivos intrínsecos (vocación y ampliar completar estudios), mientras que para los motivos extrínsecos (en concreto salida profesional y realizar estudios no deseados como primera opción) los que lo siguieron presentan un menor nivel de satisfacción.

- Finalmente, tanto en Sociales como en Técnicas, la vocación es el único motivo que marca diferencias significativas, quienes lo tuvieron en cuenta tienen un mayor nivel de satisfacción.

Al comparar la principal motivación intrínseca (vocación) con la principal extrínseca (salida profesional) dentro de cada área, y para un nivel de significación del 5\%, en todas las áreas científicas la vocación genera mayor nivel de satisfacción que la salida profesional, como se postulaba en la hipótesis H2c.

\section{Motivaciones de elección y sexo}

Todas las motivaciones presentan diferencias estadísticamente significativas a la hora de ser tomadas en cuenta entre hombres y mujeres (excepto cuando la elección no es la deseada como primera opción). Los motivos extrínsecos tienen mayor presencia entre los hombres, mientras que las mujeres se encuentran más motivadas por la vocación. La excepción supone el hecho de ampliar conocimientos que es más tenido en cuenta por los hombres.

Por otro lado, las mujeres manifiestan una mayor satisfacción, como cabría esperar de quienes se orientan más por motivaciones intrínsecas. Esto concuerda con los estudios que han puesto de relieve esta relación más fuertemente vocacional entre las mujeres (Midgley et al., 2001; Barberá, 2004). Por separado, tanto en hombres como en mujeres, quienes eligieron por motivos intrínsecos presentan mayor satisfacción. Estas diferencias no son significativas para el caso de los motivos extrínsecos, con la excepción de cuando se eligió en negativo (no la opción preferida) que conduce a una menor satisfacción. Por tanto se encuentra apoyo para las hipótesis H3a y H3b. 
Tabla 7. Motivos de elección por sexo (porcentaje sobre el total) y media de satisfacción por sexo

\begin{tabular}{lccc}
\hline Motivos intrínsecos & Elección & Mujer & Hombre \\
\hline Vocación (0.00) & Sí & 58.9 & 54.9 \\
\hline Ampliar y completar conocimientos (0.03) & Sí & 16.7 & 18.3 \\
\hline Motivos extrínsecos & Sí & 26.8 & 34.6 \\
\hline Salidas profesionales $(0.00)$ & Sí & 9.0 & 10.5 \\
\hline Recomendación de otras personas $\left(0.01^{*}\right)$ & Sí & 6.1 & 6.0 \\
\hline Imposibilidad de elegir la opción deseada (n.s.) & Sí & 5.7 & 9.7 \\
\hline Tradición familiar $(0.00)$ & Media & 3.74 & 3.57 \\
\hline Satisfacción $(0.00)$ & & & \\
\hline
\end{tabular}

Al comparar la principal motivación intrínseca (vocación) con la principal extrínseca (salida profesional) según el sexo, y para un nivel de significación del 5\%, en ambos sexos la vocación genera mayor nivel de satisfacción que la salida profesional (se confirma hipótesis $\mathrm{H} 3 \mathrm{c}$ ).

\section{Motivaciones de elección y nivel de formación de los padres}

Al considerar el nivel de formación de los padres no se presentan tantas diferencias significativas para las diferentes motivaciones. No hay diferencias en las dos principales (vocación y salida profesional). Hay diferencias para el caso de completar o ampliar estudios, curiosamente más para los que tienen padres con menor nivel de estudios. Esto puede deberse a que en un principio se eligen estudios de menor duración y después se opta por continuar un segundo ciclo. El segundo caso es el motivo de tradición familiar asociado a un mayor nivel de formación de los padres. El porcentaje de presencia de este motivo en la elección aumenta con el nivel de formación de los padres.

El nivel de satisfacción general disminuye a medida que aumenta el nivel de formación de los padres. En este sentido, la literatura no es unánime. Esta conclusión contradice a los estudios que establecen una correlación entre un mayor nivel de estudios de los padres y una mayor reafirmación con el hecho de haber realizado una carrera universitaria (Flint, 1992; Mullen et al. 2003). La presión del entorno familiar delimita la motivación y las familias sin tradición universitaria influyen en las nuevas generaciones, esto puede 
condicionar la satisfacción (Ceja, 2006). Una explicación puede deberse al hecho de que los padres que no acudieron a la universidad, transmiten la importancia a sus hijos de tener estudios superiores dado que ellos no los realizaron. Los egresados con padres con estas características otorgan una mayor importancia a la asistencia a la universidad, se produciría una especie de mitificación o hiperrealidad entre los estudiantes con el hecho de realizar y terminar estudios universitarios y encontrar satisfacción por ello.

En general, estos resultados no permiten confirmar la hipótesis H4a de existencia de diferencias entre las principales motivaciones según el nivel de formación de los padres. Sin embargo si se encuentra apoyo para la hipótesis H4b de diferencia de satisfacción según la formación de los padres (a mayor formación, menor satisfacción).

Tabla 8. Motivos de elección y nivel de formación de los padres (porcentaje sobre el total) y media de satisfacción

\begin{tabular}{lcccc}
\hline Motivos intrínsecos & Elección & $\begin{array}{c}\text { Sin } \\
\text { estudios } \\
\text { primarios }\end{array}$ & Secundarios & Superiores \\
\hline Vocación (n.s.) & Sí & 57.2 & 59.7 & 56.6 \\
\hline Ampliar y completar conocimientos (0.02) & Sí & 18.9 & 16.5 & 16.3 \\
\hline Motivos extrínsecos & Sí & 28.3 & 28.9 & 30.8 \\
\hline Salidas profesionales (n.s) & Sí & 9.9 & 8.6 & 9.8 \\
\hline Recomendación de otras personas (n.s) & Sí & 6.3 & 6.1 & 5.8 \\
\hline Imposibilidad de elegir otra (n.s.) & Sí & 3.3 & 5.0 & 11.9 \\
\hline Tradición familiar (0.00) & Media & 3.77 & 3.7 & 3.58 \\
\hline Satisfacción (0.00) & & & & \\
\hline
\end{tabular}

Dentro de cada categoría de nivel de estudios de los padres, el tener en cuenta los dos motivos intrínsecos (vocación, en las tres categorías, y ampliar/ completar estudios en las dos categorías extremas, de mayor y menor nivel de formación) genera diferencias estadísticamente significativas en el nivel de satisfacción. Mientras que elegir algo que no era lo más deseado produce menos satisfacción en las tres categorías de nivel de formación. De nuevo al comparar la principal motivación intrínseca (vocación) con la principal extrínseca (salida profesional), según el nivel de formación de los padres para todas las categorías, la vocación genera mayor satisfacción que la salida profesional. Así, no se rechaza la hipótesis H4c. 


\section{Perfil del egresado con una mayor satisfacción}

Para establecer el perfil de la satisfacción con las variables anteriormente utilizadas, se ha realizado un árbol de segmentación jerárquica. Para ello se utiliza el modelo de crecimiento CHAID; como criterio para la partición se estableció un mínimo de 100 casos para el nodo parental y de 50 para el nodo filial, y un valor mínimo del $5 \%$ en la prueba de Chi-cuadrado. Los resultados se muestran en la Figura 2.

El sexo es la característica que discrimina más el nivel de satisfacción, seguida del nivel de estudios de los padres y luego los motivos de elección, uno intrínseco (vocación) y otro extrínseco (recomendación de terceros). La satisfacción mayor corresponde a mujeres con padres sin estudios superiores, tanto si eligen por vocación (nodo 11, el grupo más numeroso con un $24.1 \%$ muestral y con la mayor satisfacción, 3.84) como si no eligen por vocación $(10,16.3 \%$, satisfacción del 3.73).

Sigue con satisfacción por encima de la media general, las egresadas cuyos padres tienen estudios superiores y que estaban motivadas por la vocación. Representan el 13.9\% y una media de 3.71 (nodo 9). Y el grupo de egresados con los padres de menor nivel de formación, que siguieron recomendación de terceras personas (11.1\%, nodo 14). Los menores niveles de satisfacción se dan entre hombres cuyos padres tenían el menor nivel de formación y que siguieron la recomendación de terceros (nodo 15 con pocos casos, 1.3\%) y el grupo con características opuestas cuyos padres tenían estudios superiores pero que no tuvieron en cuenta la recomendación de terceros (12).

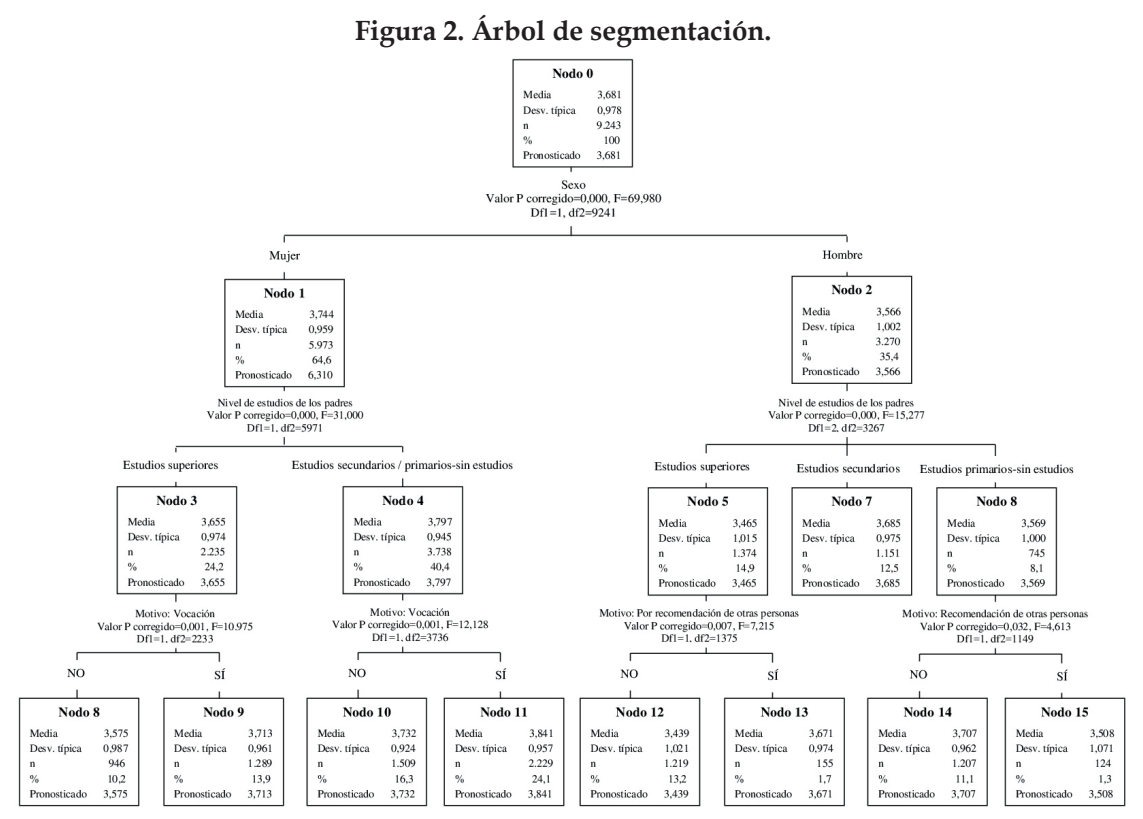




\section{Conclusiones}

Tras analizar algunos de los factores más relevantes que intervienen en el proceso de evaluación del paso por la universidad por parte de los egresados, se tiene una medida de su importancia en la satisfacción general de los mismos.

En primer lugar, los resultados alcanzados constatan que la decisión de los estudios a cursar es un compendio entre motivos intrínsecos y extrínsecos (Faye y Sharpe 2008; Arquero et al, 2009), pero principalmente debido a la vocación y las salidas profesionales. La elección de la carrera por motivos intrínsecos proporciona mayor satisfacción. La vocación o el deseo de ampliar conocimientos reafirma la elección realizada por los estudiantes, mientras que los motivos como la tradición familiar, las salidas profesionales y la imposibilidad de elegir otra dan lugar a una menor satisfacción. Únicamente la recomendación de terceras personas no da lugar a diferencias significativas.

En segundo lugar, atendiendo a las diferencias de motivaciones según área de conocimiento, en todos los ámbitos científicos la vocación es el principal aspecto que se tiene en cuenta. Sin embargo, cada área está condicionada por criterios diferentes a la hora de seleccionar sus estudios:

- Humanidades es la que está más influenciada por los motivos intrínsecos de vocación y ampliación de conocimientos. Este resultado es acorde por investigaciones anteriores (Zeegers, 2004; Castro y Egaña, 2008).

- En Ciencias de la Salud es donde más influye la tradición y la recomendación familiar.

- Enseñanzas Técnicas está más influenciada por las salidas profesionales y la recomendación de otras personas. Por lo tanto, es la que tiene más en cuenta los motivos extrínsecos.

- Ciencias Sociales está altamente influenciado por las salidas laborales.

- Ciencias Experimentales es la más condicionada por la imposibilidad de elegir otra carrera.

En todas las áreas, cuando se tienen en cuenta los aspectos intrínsecos, aumenta la satisfacción.

En cuanto al sexo, las mujeres tienen más en cuenta la vocación a la hora de elegir sus estudios, mientras que los hombres se encuentran más influenciados por el hecho de ampliar conocimientos. Sin embargo, en todos los motivos extrínsecos hay una mayor influencia en los hombres. Las mujeres tienen una mayor satisfacción con la universidad precisamente por el hecho de ser más vocacionales y estar menos influenciadas por motivaciones extrínsecas. 
El nivel de estudios de los padres tiene una especial incidencia y condiciona de manera importante la satisfacción. Los resultados hallados establecen que los alumnos cuyos padres tienen un nivel educativo bajo tienen una mayor satisfacción. Estos padres generan unas especiales expectativas al acudir a la universidad. Posiblemente los egresados de padres con menor formación tengan una menor disponibilidad de información o tengan unas expectativas mayores al no tener un entorno familiarizado con la formación universitaria, mientras que los egresados cuyos padres tenían estudios superiores pueden mostrarse más críticos ya que disponen de más información para comparar y, en consecuencia, mostrar menor nivel de satisfacción.

Las motivaciones intrínsecas marcan la diferencia en los niveles de satisfacción, además de ser determinantes en la decisión por el estudiante; es algo a tener muy presente en el asesoramiento de orientadores, familiares y terceros, así como en las estrategias de comunicación de las universidades.

De todo lo anterior cabe extraer implicaciones para las universidades y autoridades públicas que quieran fomentar determinadas vocaciones, como es el caso de la promoción de las materias stem (Science, Technology, Eengineering y Mathematic) de lo que ya hay diversas iniciativas en la Unión Europea y Estados Unidos como forma de abordar los nuevos retos económicos y sociales. La promoción de estas materias debe basarse en motivaciones intrínsecas y en períodos tempranos de la educación secundaria, no en el último año cuando ya la elección está bastante delimitada. En la misma línea, se debe actuar para promover los estudios en enseñanzas técnicas entre las mujeres, un campo claramente descompensado por motivos de sexo.

El nivel de formación de los padres no es tan determinante en la satisfacción final. La cantidad de información existente por diferentes vías (personales, on line y off line) diluye tal efecto.

Finalmente, recurrir a la identificación de grupos por niveles de satisfacción permite diseñar una atención más cercana y adaptada a las características personales y sus motivaciones. Esto constituye la base para acciones de comunicación adecuadas para cada uno de los diferentes segmentos mejorando la atención, el seguimiento y el asesoramiento general al estudiante y para los diferentes momentos del proceso de enseñanza-aprendizaje. Tal como afirman Maringe y Gibbs (2009), la segmentación en el contexto de la educación superior permite optimizar esfuerzos y atraer a nuevos estudiantes.

Entre tales acciones a realizar, las universidades pueden llevar a cabo programas de orientación previa a la etapa estudiantil en los institutos y centros educativos, además de charlas, jornadas (por ejemplo, de puertas abiertas) o campus de verano para estimular-descubrir vocaciones, lo que tiene un valor especial para quienes no tienen un contexto familiar de conexión con la edu- 
cación superior. Por supuesto, ante la elección de estudios, los estudiantes deben de tener accesibles estudios e informes (sobre nivel de satisfacción, empleabilidad o seguimiento de egresados) que les ayuden a un proceso de elección documentado y racional. Así, por ejemplo, podrán comprender el impacto de los diferentes motivos de elección en la evaluación a posteriori y su influencia en el largo plazo.

\section{Referencias}

Ahmed, Kamran; Alam, Kazi Feroz y Alam, Manzurul (1997). An empirical study of factors affecting accounting students' career choice in New Zealand. Accounting Education, 6 (4), 325-335.

Arquero, José L.; Byrne, Marann; Flood, Barbara y González, Jose María (2009). Motives, expectations, preparedness and academic performance: a study of students of accounting at a spanish university. Revista de Contabilidad, 12 (2), 279-299.

Auyeung, Pak y Sands, John (1997). Factors influencing accounting students' career choice: a cross-cultural validation study. Accounting Education, 6 (1), 13-23.

Barberá, Ester (2004). Psicología y Género. Madrid: Ediciones Pearson.

Byrne, Marann y Flood, B. (2005). A study of accounting students' motives, expectations and preparedness for higher education. Journal of Further and Higher Education, 29 (2), 111-124.

Byrne, Marann y Flood, Barbara (2007). Exploring the antecedents of learning approaches: a study of international business students. International Journal of Management Education, 6 (2), 44-62.

Byrne, Marann, Willis, Pauline y Burke, John (2012). Influences on school leavers' career decisions-Implications for the accounting profession. The International Journal of Management Education, 10 (2), 101-111.

Castro, M. y Egaña, E. (2008). Adolescentes y elección profesional: factores que influyen en la decisión. CEPAL. I Congreso Latinoamericano de jóvenes investigadores en juventud "Jóvenes en el saber". Santiago de Chile.

Ceja, Miguel (2006). Understanding the role of parents and siblings as information sources in the college choice process of Chicana students. Journal of College Student Development, 47 (1), 87-104.

Condry, John (1987). Enhancing motivation: A social developmental perspective. Advances in motivation and achievement: Enhancing motivation, 5, 23-49.

Deci, Edward L., \& Ryan, Richard M. (2000). The" what" and" why" of goal pursuits: Human needs and the self-determination of behavior. Psychological inquiry, 11(4), 227-268.

Duart, Pura; Quiñones, Olga y Tobara, Andreu (1993) L'elecció d'estudis universitaris; opinions, representacions i valors sobre les preferències universitàries dels estudiants valencians. Valencia: Universidad de Valencia. Valencia. 
Eccles, Jacquelynne S.; Barber, Bonnie y Jozefowicz, Debra (1999). Linking gender to educational, occupational, and recreational choices: Applying the Eccles et al. model laof achievement-related choices, Washington: American Psychological Association, pp. 153-192.

Faye, Cathy y Sharpe, Donald (2008). Academic motivation in university: The role of basic psychological needs and identity formation. Canadian Journal of Behavioural Science/Revue canadienne des sciences du comportement, 40 (4), 189-199.

Flint, Thomas A. (1992). Parental and planning influences on the formation of student college choice sets. Research in Higher Education, 33 (6), 689-708.

Gámez, Elena \& Marrero, Hipólito (2003). Metas y motivos en la elección de la carrera universitaria: Un estudio comparativo entre psicología, derecho y biología. Anales de psicología, 19 (1), 121-131.

García García, Luis Alberto; Martín Cabrera, Eduardo y Capote, Morales, María Carmen (1994). Perfiles Motivacionales en la elección de carrera universitaria. Revista Galega de Psicopedagogía, 10-11 (7), 203-218.

Gatica Patricio; Vargas, Rodrigo; Granada, Maribel y Ramírez, Luis (2012). Convergencia educativa, 1 (2), 71-85.

González López, Ignacio (2005). Motivación y actitudes del alumnado universitario al inicio de la carrera. ¿Varían al egresar? Revista electrónica de Investigación Psicoeductativa y Psicopedagógica, 5-3 (1), 35-56.

Gul, Ferdinand; Huang, Allen \& Subramaniam, Nava (1992). Cognitive style as a factor in accounting students' perceptions of career-choice factors. Psychological Reports, 71(3 suppl), 1275-1281.

Hearn, James C. (1988). Attendance at higher-cost colleges: Ascribed, socioeconomic, and academic influences on student enrollment patterns. Economics of education review, 7 (1), 65-76.

Law, Philip y Yuen, Desmond (2012). A multilevel study of students' motivations of studying accounting: Implications for employers. Education+ Training, 54 (1), 50-64.

López-Bonilla, Jesús Manuel; Barrera Barrera, Ramón; Rodríguez Serrano, María Ángeles; López-Bonilla, Luis Miguel; Palacios Florencio, Beatriz; Reyes Rodríguez, María Carmen \& Sanz Altamira, Borja (2012). Reasons which influence on the students' decision to take a university course: differences by gender and degree. Educational Studies, 38 (3), 297-308.

Luque Martínez, Teodoro (Coord). (2015). The University of Granada in Light of its V Centenary. "Reflections on the Future of the University. Granada: Editorial Universidad de Granada.

Teodoro Luque Martínez; Salvador del Barrio García; Juan Sánchez Fernández; José Angel Ibáñez Zapata; Luis Doña Toledo (2013). Estudio de Egresados de la Universidad de Granada. Año 2009. Granada: Editorial Universidad de Granada

Teodoro Luque Martínez; Salvador del Barrio García; Juan Sánchez Fernández; José Angel Ibáñez Zapata; Luis Doña Toledo (2014). Estudio de Egresados de la Universidad de Granada. Año 2010. Granada: Editorial Universidad de Granada. 
Teodoro Luque Martínez; Salvador del Barrio García; Juan Sánchez Fernández; José Angel Ibáñez Zapata; Luis Doña Toledo (2015). Estudio de Egresados de la Universidad de Granada. Año 2011. Granada: Editorial Universidad de Granada.

Maines, David R. (1983). A theory of informal barriers for women in mathematics. Annual meeting of the American Educational Hesearch Association, Montreal.

Martín-Cobos, Manuel, \& Salas, Manuel (2006). La demanda de educación superior: un análisis microeconómico con datos de corte transversal. Revista de educación, (339), 637-660.

McDonough, Patricia (1997). Choosing colleges: How social class and schools structure opportunity. New York: suny Press.

Midgley, Carol; Kaplan, Avi; y Middleton, Michael (2001). Performance-approach goals: Good for what, for whom, under what circumstances, and at what cost? Journal of Educational Psychology, 93 (1), 77-86.

mecd. (2015). Datos y cifras del sistema universitario español.

Mingo, Araceli (2016). "¡Pasen a borrar el pizarrón!" Mujeres en la universidad. Revista de la Educación Superior, 45(178), 1-15.

Ann L. Mullen, Kimberly A. Goyette and Joseph A. Soares

Mullen, Ann L.; Goyette, Kimberly A. y Soares, Joseph A. (2003). Who goes to graduate school? Social and academic correlates of educational continuation after college. Sociology of Education, 17 (2), 143-169.

Muñoz, María Teresa y Mullet, Etienne (1990). Los determinantes de las preferencias profesionales de los adolescentes. Evaluación Psicológica, 6, 155-170.

Neave, Guy y Veiga, Amelia (2013). The Bologna Process: inception, 'take up' and familiarity. Higher Education, 66 (3), 59-77.

ocde (2014), Education at a Glance 2013: OECD.

Paa, Heidi K. y McWhirter, Ellen Hawley (2000). Perceived influences on high school students' current career expectations. The Career Development Quarterly, 49 (1), 29-44.

Palos, Ramona y Drobot, Loredana (2010). The impact of family influence on the career choice of adolescents. Procedia-Social and Behavioral Sciences, 2 (2), 3407-3411.

Paolillo, Joseph G. P. y Estes, Ralph W. (1982). An Empirical Analysis of Career Choice Factors Among Accountants, Attorneys, Engineers, and Physicians. The Accounting Review, 54 (4), 785-794.

Paulsen, Michael B. y Gentry, James A. (1995). Motivation, learning strategies, and academic performance: a study of the college finance classroom. Financial Practice and Education, 5 (1), 78-90.

Phillips, Susan D.; Christopher-Sisk,Erin K. y Gravino, Kristine L. (2001). Making career decisions in a relational context. The Counseling Psychologist, 29 (2), 193-214.

Price, If; Matzdorf, Fides, Smith, Louise, \& Agahi, Helen (2003). The impact of facilities on student choice of university. Facilities, 21(10), 212-222.

Rivas, Francisco (1990). La elección de estudios universitarios. Un sistema de asesoramiento universitario basado en indicadores vocacionales eficaces. Madrid. Consejo de Universidades. 
Sáinz, Milagros; López-Sáez, Mercedes y Lisbona, Ana (2004) Expectativas de rol profesional de mujeres estudiantes de carreras típicamente femeninas o masculinas. Acción Psicológica, 3, 111-123.

Schnabel, Kai U.; Alfeld, Corinne; Eccles, Jacqueline S.; Köller, Olaf y Baumert, Jürgen. (2002). Parental influence on students' educational choices in the United States and Germany: Different ramifications - Same effect? Journal of Vocational Behavior, 60 (2), 178-198.

Seymour, Daniel T. (1993). On Q: Causing Quality in Higher Education. Oryx. Phoenix, A.Z.: McMillan.

Sewell, William H., \& Shah, Vimal P. (1968). Social class, parental encouragement, and educational aspirations. American Journal of Sociology, 73(5), 559572.

Shanka, Tekle; Quintal, Vanessa y Taylor, Ruth (2006). Factors influencing international students' choice of an education destination-A correspondence analysis. Journal of Marketing for Higher Education, 15 (2), 31-46.

Silverstone, Rosalie y Williams, Allan (1979). Recruitment, training, employment and careers of women chartered accountants in England and Wales. Accounting and Business Research, 9 (34), 105-122.

Sirvanci, Mete (1996). Are students the true customers of higher education? Quality Progress, 29 (10), 99-102

Solinas, Giuliana; Masia, María Dolores; Maida, Giorgio y Muresu, Elena (2012). What Really Affects Student Satisfaction? An Assessment of Quality through a University-Wide Student Survey. Creative Education, 3 (1), 37-40.

Sugahara, Satoshi y Boland, Greg (2010). The role of cultural factors in the learning style preferences of accounting students: a comparative study between Japan and Australia. Accounting Education: an international journal, 19 (3), 235-255.

Tan, Lin Mei y Laswad, Fawzi (2006). Students' beliefs, attitudes and intentions to major in accounting. Accounting Education: an international journal, 15 (2), 167-187.

Tang, Mei Fouad, Nadya A. Smith, Philip L.

Tang, Mei; Fouad, Nadya A. y Smith, Philip L. (1999). Asian Americans' career choices: A path model to examine factors influencing their career choices. Journal of Vocational Behavior, 54 (1), 142-157.

Vrontis, Demetris; Thrassou, Alkis \& Melanthiou, Yioula (2007). A contemporary higher education student-choice model for developed countries. Journal of Business Research, 60(9), 979-989.

Wardley, Leslie J., Bélanger, Charles H. y Leonard, Valorie M. (2013). Institutional commitment of traditional and non-traditional-aged students: a potential brand measurement? Journal of Marketing for Higher Education, 23 (1), 90112.

Zeegers, Peter (2004). Student learning in higher education: A path analysis of academic achievement in science. Higher Education Research \& Development, 23 (1), 35-56.

Zellweger, Thomas; Sieger, Phillip y Halter, Frank (2011). Should I stay or should I go? Career choice intentions of students with family business background. Journal of Business Venturing, 26 (5), 521-536. 